Published in final edited form as:

J Phys Chem Lett. 2021 October 28; 12(42): 10295-10303. doi:10.1021/acs.jpclett.1c02155.

\title{
Making Nitronaphthalene Fluoresce
}

\author{
Katarzyna Rybicka-Jasińska+, \\ Department of Bioengineering, University of California, Riverside, California 92521, United \\ States; Present Address: Institute of Organic Chemistry, Polish Academy of Sciences, Warsaw, \\ Poland
}

\section{Eli M. Espinoza ${ }^{+}$,} Department of Chemistry, University of California, Riverside, California 92521, United States; Present Address: College of Bioengineering, University of California, Berkeley, CA 94720, USA

John A. Clark,

Department of Bioengineering, University of California, Riverside, California 92521, United States

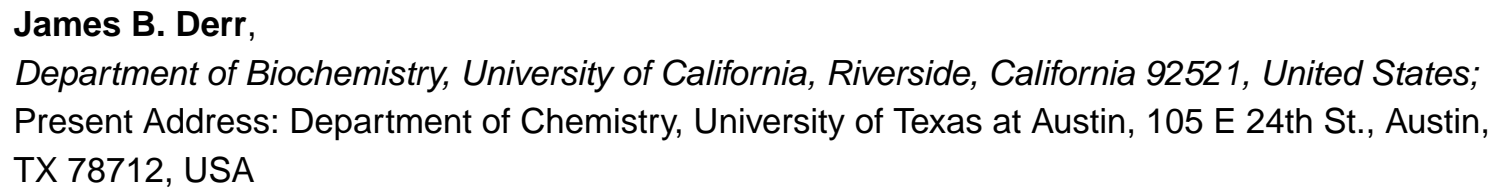

\section{Gregory Carlos,}

Department of Biology, University of California, Riverside, California 92521, United States

\section{Maryann Morales,} Department of Chemistry, University of California, Riverside, California 92521, United States; Present Address: Division of Chemistry and Chemical Engineering, California Institute of Technology, Pasadena, CA 91125, USA

\section{Mimi Karen Billones,}

Department of Biology, University of California, Riverside, California 92521, United States;

Present Address: Department of Biochemistry, University of 452 California, Riverside, California 92521, USA

\section{Omar O'Mari,}

Department of Bioengineering, University of California, Riverside, California 92521, United States

\section{Hans Ågren,}

Department of Physics and Astronomy, Uppsala University, SE-751 20 Uppsala, Sweden

\section{Glib V. Baryshnikov,}

Corresponding Authors: Hans Ågren - Department of Physics and Astronomy, Uppsala University, SE-751 20 Uppsala, Sweden; hagren@ kth.se, Glib V. Baryshnikov - Laboratory of Organic Electronics, Department of Science and Technology, Linköping University, SE-60174 Norrköping, Sweden; glib.baryshnikov@liu.se, Valentine I. Vullev - Department of Bioengineering, University of California, Riverside, California 92521, United States; Department of Chemistry, Department of Biochemistry, and Materials

Science and Engineering Program, University of California, Riverside, California 92521, United States; vullev@ucr.edu.

${ }^{+}$Author Contributions

K.R.J. and E.M.E. contributed equally.

The authors declare no competing financial interest. 
Laboratory of Organic Electronics, Department of Science and Technology, Linköping University, SE-60174 Norrköping, Sweden

\section{Valentine I. Vullev}

Department of Bioengineering, University of California, Riverside, California 92521, United States; Department of Chemistry, Department of Biochemistry, and Materials Science and Engineering Program, University of California, Riverside, California 92521, United States

\section{Abstract}

Nitroaromatic compounds are inherently nonfluorescent, and the subpicosecond lifetimes of the singlet excited states of many small nitrated polycyclic aromatic hydrocarbons, such as nitronaphthalenes, render them unfeasible for photosensitizers and photo-oxidants, despite their immensely beneficial reduction potentials. This article reports up to a 7000-fold increase in the singlet-excited-state lifetime of 1-nitronaphthalene upon attaching an amine or an $\mathrm{N}$-amide to the ring lacking the nitro group. Varying the charge-transfer (CT) character of the excited states and the medium polarity balances the decay rates along the radiative and the two nonradiative pathways and can make these nitronaphthalene derivatives fluoresce. The strong electron-donating amine suppresses intersystem crossing (ISC) but accommodates CT pathways of nonradiate deactivation. Conversely, the $\mathrm{N}$-amide does not induce a pronounced CT character but slows down ISC enough to achieve relatively long lifetimes of the singlet excited state. These paradigms are key for the pursuit of electron-deficient ( $n$-type) organic conjugates with promising optical characteristics.

\section{Graphical Abstract}

bright field
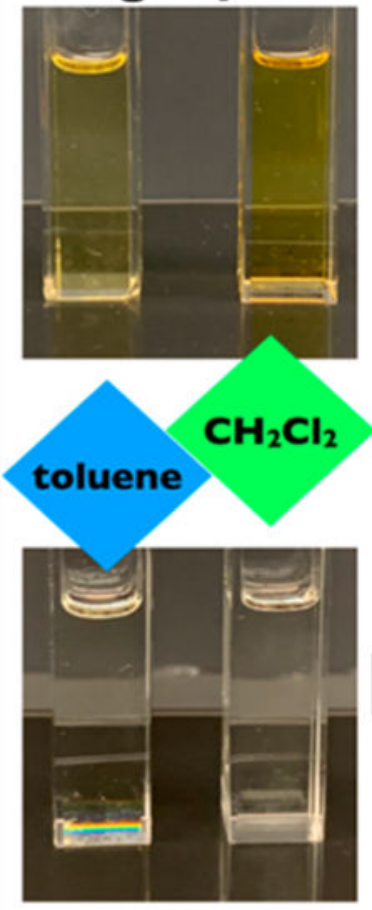

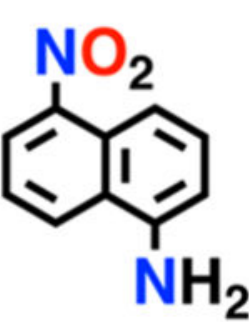

fluorescence

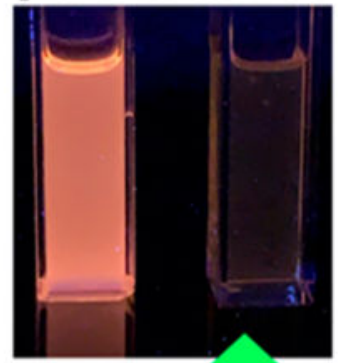

$\mathrm{CH}_{2} \mathrm{Cl}_{2}$

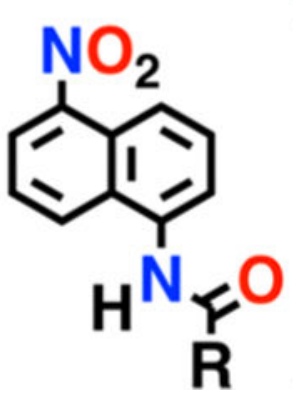

toluene

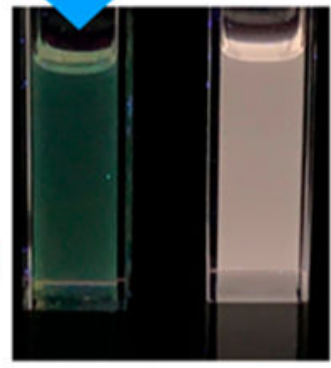


Organic aromatic compounds are building blocks for a wide variety of $p$-type materials and potent photo-reductants. Conversely, $n$-type organic conjugates are few and far between. ${ }^{1-9}$ The electron-withdrawing strength of nitro groups makes them promising substituents for attaining such $n$-type systems. Nitro groups induce positive shifts in the reduction potentials of polycyclic aromatic hydrocarbons, such as pyrene, that exceed $1 \mathrm{~V} \cdot{ }^{10-14}$ The shifts induced by nitrile and carbonyls range between 0.2 and $0.7 \mathrm{~V} .{ }^{15,16}$

Optically excited states of most nitro-organic compounds, however, undergo fast nonradiative decays. That is, nitro groups are efficient fluorescence quenchers, rendering these compounds unfeasible for a wide range of applications such as photosensitization and photo-oxidation. Therefore, understanding how to make nitro polycyclic aromatics fluoresce is crucial for photonics, electronics, and energy science.

Nitro groups usually induce high densities of $\pi \pi^{*}$ and $n \pi^{*}$ states with different multiplicity, providing pathways for efficient intersystem crossing (ISC), i.e., triplet formation. ${ }^{17-21}$ Introducing a charge-transfer (CT) character in the singlet or triplet excited states can decrease the spin-orbit coupling (SOC) between them and reduce the ISC rates. ${ }^{22}$ Many of the fluorescent nitro compounds, indeed, carry electron-donating moieties ${ }^{21,23-26}$ or are built on large polycyclic cores that are electron donors themselves. ${ }^{27}$ A nitro-induced strong CT character also leads to nonfluorescent excited states with negligible overlaps between their natural transition orbitals (NTOs), resulting in slow radiative decay. ${ }^{20,28}$ Furthermore, medium polarity stabilizes such CT states, bringing them close to the ground state, which favors internal conversion (IC), and enhances the nonradiative decay rates. ${ }^{20}$ As we recently reported, frustrated photochemistry, involving the formation of intramolecular covalent bonds in the excited state that break upon relaxation to the ground state, is another pathway of fluorescence quenching induced by the strongly polarizing capabilities of nitro groups. ${ }^{29}$ This conundrum of excited-state CT that reduces ISC, while augmenting IC deactivation via back CT or frustrated photochemistry, raises the question of how much CT character is too much.

Nitroaromatics actually fluoresce. ${ }^{30}$ With fluorescence quantum yields $\left(\phi_{\mathrm{f}}\right)$ smaller than $10^{-4}$, and singlet-excited-state lifetimes $(\tau)$ shorter than a few picoseconds, however, nitroaromatics are practically nonfluorescent. Nitronaphthalenes (NNs) are among the smallest electron-deficient aromatic compounds, exhibiting ISC on time scales of $\sim 100$ $\mathrm{fs},{ }^{31}$ which has made them a classic illustration of how a nitro group induces efficient triplet formation. Therefore, making NNs fluorescent is as challenging as it is crucial for paving pathways to electron-deficient nitroaromatics with long-lived singlet excited states.

In the 1960s, Corkill and Graham-Bryce reported the emission of amino derivatives of mono- and dinitronaphthalenes in frozen alcohols. ${ }^{32}$ These compounds are weakly emissive even in a solid media at cryogenic temperatures, and 2-, 4-, and 5-amino-1-nitronaphthalene exhibit detectable phosphorescence, rather than fluorescence, under such conditions, ${ }^{32}$ which is common for nitronaphthalenes. ${ }^{33}$ A procedure for the synthesis of 3,7-diamino-1,5dinitronaphthalene reports recrystallization leading to the formation of fluorescent dark prisms, but without any further photophysical studies and proofs. ${ }^{34}$ Attaching nitrophenyl groups to naphthalene offers weakened electronic coupling between the nitro groups and the 
polycyclic hydrocarbon core, resulting in fluorescence quantum yields between about 0.01 and 0.1 and excited-state lifetimes between abut 0.2 and $2 \mathrm{~ns} .{ }^{35}$ Push-pull conjugates of naphthalene oligomers with an amine and a nitro group at their termini show a reasonable $\phi_{\mathrm{f}}$ of about 0.01 only when their size extends to four units, thus ensuring weakened electronic coupling of the terminal nitro group with the NTOs for the radiative deactivation. ${ }^{36}$ Such strategies of weakening the electronic coupling with the nitro groups in order to suppress their detrimental effects on the photophysics also diminish the benefits from their electronwithdrawing strength for the electronic properties of the chromophores. Furthermore, the added conformational degrees of freedom from biaryl or oligomethine linkers with nitrophenyls induce alternative IC pathways for efficient nonradiative decay through conical intersection between the potential-energy surfaces of the $\mathrm{S}_{1}$ and $\mathrm{S}_{0}$ states (involving back CT or frustrated photochemistry, for example), as the strong dependence of $\phi_{\mathrm{f}}$ on the medium viscosity reveals. ${ }^{29,37,38}$

Hence, it is crucial to pursue organic dyes with the nitro groups directly attached to their chromophore cores. As Peon et al. demonstrated, however, placing an electron-donating group at position 4 of 1-nitronaphthelene (1, Chart 1$)$ only marginally improves its fluorescence. The 4-amino and 4-methoxy derivatives of $\mathbf{1}$ show biexponential fluorescence decays with major components of $\sim 0.1 \mathrm{ps}$ and minor ones of $\sim 2$ ps or less. ${ }^{43}$ Placing electron-donating substituents on the ring that has the nitro group, therefore, is not truly beneficial for extending the lifetimes of the singlet-excited states of NNs. On the other hand, will attaching electron-donating substituents to the ring without the nitro group favorably impact the NN photophysics? This study pursues answers to this question by focusing on $\mathrm{NN}$ with electron-donating groups attached to position 5 (Chart 1).

Avoiding nitro groups all together has led to some broadly used biological photoprobes based on naphthalene push-pull configuration that manifest fluorescence with high $\phi_{\mathrm{f}}$ from states with a pronounced CT character. Carbonyl groups tend to drive triplet formation in quantitative yields, and alkanionyls of naphthalene are no exception. Inducing a CT character in the singlet excited state of such compounds can suppress their propensity for ISC and result in strongly fluorescent dyes, such as 1-[6-(dimethylamino)-2naphthalenyl]-1-propanone (pro-dan). ${ }^{44-46}$ 5-(Dimethylamino)naphthalene-1-sulfonamide (dansylamide) presents another example of an immensely popular fluorescent photoprobe with a naphthalene push-pull architecture (Chart 1). ${ }^{47,48}$ Carbonyls and sulfonamides, on the other hand, are considerably weaker electron-withdrawing substituents than the nitro group, ${ }^{49}$ and with reduction potentials of about $-2.1 \mathrm{~V}$ vs SCE, prodan and dansylamide are pronouncedly weaker electron acceptors than nitronaphthalenes (Chart 1). ${ }^{41,42}$

Therefore, making nitronaphthalenes and other nitroaromatics fluoresce is still a key challenge for molecular photonics. Attaching nitro groups directly to the condensed polycyclic cores of the chromophores is most beneficial for the pursuit of electron-deficient nitroaromatics. Concurrently, electron-donating substituents can prevent the nonradiative deactivation that the nitro groups induce. Placing these electron-donating substituents on the same ring where the nitro group is, however, does not prove beneficial for NNs. 
Herein, we show dramatic improvement of NN fluorescence by adding an electron-donating group to position 5, i.e., to the ring of $\mathbf{1}$ that does not bear the nitro group. It induces up to a 7000-fold increase in $\tau$. Polar media enhances the nonradiative decay of the 5-amino NN (2, Chart 1). As a weaker electron donating group than the amine, an $\mathrm{N}$-amide at position 5 $\left(3\right.$, Chart 1) substantially decreases the polarity dependence of $\tau$ and $\phi_{\mathrm{f}}$. Therefore, attaching the auxiliary electron-donating substituents to rings without nitro groups is essential for extending the lifetimes of the singlet-excited states of such nitroaromatic chromophores.

The nitro group causes about a 1.4-V positive shift in the reduction potential of naphthalene (Chart 1, Figure 1), which is enormous, making 1 a potent electron acceptor and a photooxidant in its $\mathrm{S}_{1}$ state. Incorporation of an amine or an $N$-amide alters $E_{\mathrm{Xl}}^{(1 / 2)} \bullet-$ by less than $0.1 \mathrm{~V}$ (Chart 1, Figure 1).

While the electron-donating substituents at position 5 have a negligible effect on the potency of $\mathbf{1}$ as an electron acceptor, they strongly affect its photophysics. $\mathbf{2}$ absorbs around $400 \mathrm{~nm}$ and shows a broad fluorescence band that shifts from 600 to $700 \mathrm{~nm}$ upon increasing solvent polarity (Figure 2a). The $400 \mathrm{~nm}$ band, originating from amine-nitro push-pull interactions, is weakly solvatochromic with extinction coefficients 4-to-6 fold smaller than those for 4-amino NN (Figure 3a, Table 1), ${ }^{53}$ i.e., moving the amine from position 4 to 5 weakens its coupling with the nitro group.

Even at micromolar concentrations, $\mathbf{2}$ aggregates. These aggregates exhibit huge Stokes' shifts $(\Delta \boldsymbol{\varepsilon})$ that exceed $1 \mathrm{eV}$. Decreasing the concentration of $\mathbf{2}, C(\mathbf{2})$, induces a growth of an emission band at $\sim 450 \mathrm{~nm}$, ascribed to the monomer fluorescence (Figure $2 \mathrm{~b}, \mathrm{c}$ ). At concentrations smaller than about $100 \mu \mathrm{M}$, the monomer and the aggregates of $\mathbf{2}$ contribute to its absorption spectra. The excitation spectra for the two emission bands greatly overlap. The absorption, however, closely resembles the excitation spectrum for the bathochromic emission (Figure $2 \mathrm{~b}, \mathrm{c}$ ). Within the working micromolar concentration ranges, therefore, 2 exists predominantly as an aggregate.

For toluene, the $\phi_{\mathrm{f}}$ of $\mathbf{2}$ is $\sim 0.01$ and $\tau \approx 0.7 \mathrm{~ns}$. Both drastically decrease with an increase in solvent polarity. It reveals a polarity-induced increase in nonradiative decay constant $\left(k_{\mathrm{nd}}\right)$ from $10^{9}$ to $10^{11} \mathrm{~s}^{-1}$, while the radiative-decay rate constant $\left(k_{\mathrm{f}}\right)$ remains around $10^{7} \mathrm{~s}^{-1}$ (Figure 3c,d).

Replacing the amine with an $\mathrm{N}$-amide, $\mathbf{3}$, yields similar spectral features that are hypsochromically shifted by $\sim 50 \mathrm{~nm}$, vs those of $\mathbf{2}$ (Figure $2 \mathrm{~d}-\mathrm{f}$ ). $\mathbf{3}$ exhibits weaker monomer emission than $\mathbf{2}$. Attached via their carbonyl carbons, amides can serve as weakly electron-withdrawing substituents. ${ }^{12,23}$ When attached via their nitrogens, as in $\mathbf{3}$, the same amides act as electron-donating substituents. ${ }^{12,23}$ Unlike $\mathbf{2}$, the aggregate band of $\mathbf{3}$ in low-polarity solvents shows concentration dependence.

The most important feature of the $N$-amide substituent is the suppression of the solvent dependence of $\phi_{\mathrm{f}}$ and $\tau$ (Figure 3c, Table 1). For toluene, a $\phi_{\mathrm{f}}$ of $\mathbf{3}$ is more than an order of magnitude smaller than that of $\mathbf{2}$ and $k_{\mathrm{f}}(3)<k_{\mathrm{f}}(2)$ while $k_{\mathrm{nd}}(3) \approx k_{\mathrm{nd}}(2)$. An increase in medium polarity does not affect the $k_{\mathrm{f}}$ of $\mathbf{3}$. Solvent changes from toluene to $\mathrm{CH}_{2} \mathrm{Cl}_{2}$ slightly 
diminish $k_{\text {nd }}(3)$. A further increase in the polarity to $\mathrm{CH}_{3} \mathrm{CN}$ induces a 4-fold increase in $k_{\text {nd }}(3)$ (Figure $3 d$, Table 1 ). Hence, two opposing polarity effects are at play for 3.

The relatively small polarity dependence of the Stokes' shifts (Figure 3a,b) reveals that the emissive $S_{1}$ states of $\mathbf{2}$ and $\mathbf{3}$ do not have much larger electric dipoles than their ground states. The polarity-induced increase in $k_{\text {nd }}$ of $\mathbf{2}$, however, suggests decay via a state with a pronounced CT character.

Following femtosecond ISC, the picosecond $\mathrm{T}_{n} \rightarrow \mathrm{T}_{1}$ relaxation of 1 leads to the rise of a $600 \mathrm{~nm}$ broad $\mathrm{T}_{1}$ transient absorption (TA) band. ${ }^{54,55}$ In contrast, photoexcitation of 2 and 3 leads to the formation of a locally excited state, $S_{1}(\mathrm{LE})$, that exhibits strong TA at 450-500 nm, along with stimulated emission (SE) at 550-650 nm (Figure 4a,b). 2 shows the rise of a weak, broad TA band around $550 \mathrm{~nm}$, which accompanies not only the decay of $\mathrm{S}_{1}$ (LE) but also a hypsochromic shift of its $450 \mathrm{~nm}$ band and a bathochromic shift of the SE. Solvent polarity enhances the formation of this broad TA feature (Figure 4a,c). For 2 in $\mathrm{CH}_{3} \mathrm{CN}$, it appears within the instrument-response time and dominates the TA spectra (Figure 4a). This $550 \mathrm{~nm}$ band differs from the long-lived $\mathrm{T}_{1} \mathrm{TA}$ and most likely corresponds to a singlet-excited state with an enhanced CT character responsible for the solvent-induced increase in $k_{\mathrm{nd}}$. For all solvents, the decay of $\mathrm{S}_{1}{ }^{(\mathrm{LE})}$ and $\mathrm{S}_{1}{ }^{(\mathrm{CT})}$ of 2 does not lead to long-lived broad TA bands characteristic for $\mathrm{T}_{1}$ absorption of NNs. Therefore, $\mathrm{S}_{1}(\mathrm{CT})$ $\rightarrow \mathrm{S}_{0}$ IC dominates the nonradiative deactivation of 2 .

For $\mathrm{CH}_{3} \mathrm{CN}$, the TA behavior of $\mathbf{3}$ is similar to that of $\mathbf{2}$ for less polar solvents (Figure 4a,b). For toluene, $\mathrm{CHCl}_{3}$, and $\mathrm{CH}_{2} \mathrm{Cl}_{2}$, however, the decay of $\mathrm{S}_{1}{ }^{(\mathrm{LE})}$ of 3 accompanies a rise of long-lived TA, ascribed to $\mathrm{T}_{1} \rightarrow \mathrm{T}_{n}$, with a relatively large amplitude, without apparent accumulation of $\mathrm{S}_{1}(\mathrm{CT})$ (Figure $4 \mathrm{~b}$ ). For nonpolar media, ISC dominates the nonradiative deactivation of $\mathbf{3}$. But even then, due to the weakly electron-donating $N$-amide, the ISC rates for $\mathbf{3}$ are orders of magnitude smaller than those for $\mathbf{1}$. A further increase in solvent polarity lowers the energy of $S_{1}(\mathrm{CT})$ of $\mathbf{3}$. It enhances the IC to $\mathrm{S}_{0}$ and $k_{\mathrm{nd}}$ of $\mathbf{3}$. Even though, at the working concentrations, these NNs exist as aggregates, our findings show important general paradigms of how to make nitroaromatics fluoresce.

To gain insight into the excited-state dynamics of the monomeric forms of $\mathbf{2}$ and $\mathbf{3}$, we resort to time-dependent density-functional theory (TDDFT) with toluene and acetonitrile solvation media as implemented by the polarizable continuum model (PCM) for optimizing the S0 and S1 structures and by the conductor-like screening model (COSMO) for calculating the transition energies, oscillator strength, and SOC matrix elements. As expected, adding electron-donating groups to $\mathrm{NN}$ increases its ground-state dipole, $\mu_{0}$, from 5.6 D for 1 to more than $7 \mathrm{D}$ for $\mathbf{2}$ and $\mathbf{3}$ in toluene (Figure 5a). While increasing the medium polarity increases the magnitudes of the dipoles due to the Onsager fields in the solvation cavities, ${ }^{56}$ the $\mu_{0}$ values of $\mathbf{1}, \mathbf{2}$, and $\mathbf{3}$ in acetonitrile show the same trends as in toluene.

Photoexcitation of 2 leads to a Franck-Condon (FC) state, $\mathrm{S}_{1}{ }^{(\mathrm{FC})}$, with a pronounced CT character. The magnitude of the dipole of this FC state, $\mu^{(\mathrm{FC}) *}$, in toluene and acetonitrile is about twice as large as $\left|\mu_{0}\right|$ (Figure 5a). The $\mathrm{S}_{1}{ }^{(\mathrm{FC})}$ state of $\mathbf{3}$ also has CT character, but not 
as pronounced as that of $\mathbf{2}$, which is consistent with the smaller electron-donating strengths of the $N$-amide in comparison to that of the amine. For $\mathbf{3}$, the ratio $\left|\mu^{(\mathrm{FC}) *} /\right| \mu_{0} \mid$ is about 1.5 for the two implemented solvation media. In contrast, $\mathbf{1}$ forms a $\mathrm{S}_{1}{ }^{(\mathrm{FC})}$ state with $\left|\mu^{(\mathrm{FC}) *}\right|>$ $\left|\mu_{0}\right|$ in acetonitrile, but in toluene $\left|\mu^{(\mathrm{FC}) *}\right| \approx\left|\mu_{0}\right|$.

The dipole moments of the optimized $\mathrm{S}_{1}$ states, $\mu^{*}$, of 2 and 3 are quite smaller than $\left|\mu^{(\mathrm{FC}) *}\right|$ (Figure 5a). This loss of CT character upon the $\mathrm{S}_{1}(\mathrm{FC}) \rightarrow \mathrm{S}_{1}$ relaxation is consistent with the experimentally observed small dependence of $\Delta \boldsymbol{\varepsilon}$ on the solvent polarity (Figure 3b); i.e., the computed $\Delta \mu=\left|\mu^{*}\right|-\left|\mu_{0}\right|$ values are about $1 \mathrm{D}$ for $\mathbf{2}$ and $\mathbf{3}$ in toluene and acetonitrile. For 2 , an increase in medium polarity enhances the charge separation in its $S_{1}$ state, which affects the oscillator strength of the $\mathrm{S}_{1} \rightarrow \mathrm{S}_{0}$ radiative transition and drastically decreases the computed rate constants, $k_{\mathrm{f}}{ }^{(\mathrm{DFT})}$, upon transition from toluene to acetonitrile (Table 1). Decreasing the electron-withdrawing strength of the group at position 5 makes $k_{\mathrm{f}}$ (DFT) invariant to solvent polarity, as the results for 3 reveal (Table 1). For comparison, the $S_{1} \rightarrow$ $\mathrm{S}_{0}$ oscillator strength and $k_{\mathrm{f}}^{(\mathrm{DFT})}$ of $\mathbf{1}$ are abysmally small, both solvating media (Figure $5 \mathrm{a}$ ), and comparable to those of $\mathbf{2}$ in acetonitrile.

These findings show the effects of the electron-donating groups on the radiative-deactivation dynamics of NN. They are consistent with the experimentally observed polarity-induced quenching of the fluorescence of $\mathbf{2}$, and not of $\mathbf{3}$. Nevertheless, fluorescence quenching results not only from a decrease in radiative decay rates but also from an increase in the rates of nonradiative deactivation. Focusing on ISC, we examine the SOC between the $\mathrm{S}_{1}$ states and the triplet ones with lower energy. While the $\mathrm{S}_{1}$ states of the three NNs are $\pi \pi^{*}$ in nature, what sets $\mathbf{2}$ and $\mathbf{3}$ apart from $\mathbf{1}$ is the symmetry of their $\mathrm{T}_{j}$ states. The nitro group in $S_{1}$ of $\mathbf{1}$ assumes symmetrically distorted pyramidal conformation regardless of the solvent polarity, which agrees well with previous DFT and ab initio studies of NNs. ${ }^{57-59}$ This distorted structure induces an $n \pi^{*}$ character into the low lying triplet states, resulting in large SOC and subpicosecond ISC (Table 1).

In contrast, the nitro group of $S_{1}$ of 2 in toluene is planar, resulting in $\pi \pi^{*}$ symmetry of the low-lying triplet states, a small SOC (Figure 5a), and a relatively slow ISC (Table 1). Implementing acetonitrile as a solvation medium, on the other hand, induces distortion of the nitro group in the $S_{1}$ state of $\mathbf{2}$, similar to what we observe for $\mathbf{1}$, and opens channels for fast ISC. Therefore, enhanced triplet formation is responsible for the polarity-induced quenching of the fluorescence of $\mathbf{2}$ in its monomeric form. Conversely, the rates of ISC mediated by the monomer of $\mathbf{3}$ in toluene and acetonitrile differ by less than a factor of $\mathbf{2}$ (Table 1). Furthermore, they are larger than those for $\mathbf{2}$ and smaller than those for $\mathbf{1}$ (Table $1)$.

The same TDDFT analysis of a noncovalent dimer of $\mathbf{2}$, i.e., $(\mathbf{2})_{2}$, reveals different trends from those for its monomer. The huge Stokes' shift places the optimized $S_{1}$ state of the dimer under its $\mathrm{T}_{2}$ one, and the small $\mathrm{S}_{1}-\mathrm{T}_{1} \mathrm{SOC}$ results in nanosecond ISC regardless of the medium polarity (Table 1). Varying the solvation media affects the structure of the dimer. The dipoles of the $\mathrm{S}_{0}, \mathrm{~S}_{1}{ }^{(\mathrm{FC})}$, and $\mathrm{S}_{1}$ states of $(\mathbf{2})_{2}$ in toluene are 2.3, 5.5, and 3.3 D, respectively, and for acetonitrile are $0.0,0.2$, and $2.4 \mathrm{D}$ (Figure $5 \mathrm{~b}$ ). That is, an increase in solvent polarity favors $\mathrm{S}_{0}$ structures with quadrupole symmetry and no net dipole moment. 
This symmetry breaks in the $S_{1}$ state of $(\mathbf{2})_{2}$, which relaxes to dipolar structures with a CT character. An increase in medium polarity increases the difference between the dipoles of the $\mathrm{S}_{0}$ and the $\mathrm{S}_{1}$ state, $\Delta \mu=\left|\mu^{*}\right|-\left|\mu_{0}\right|$. This solvent effect on the structure of the dimer and its $\Delta \mu$ further enhances the polarity-induced decrease in the energy gap between the ground and the excited state of $(\mathbf{2})_{2}$. Narrowing the $\mathrm{S}_{0}-\mathrm{S}_{1}$ energy gap can considerably enhance the IC pathway of deactivation and account for the observed polarity-induced quenching of the aggregate fluorescence of $\mathbf{2}$.

Dimerization of $\mathbf{3}$ weakens the $\mathrm{S}_{1}-\mathrm{T}_{\mathrm{j}} \mathrm{SOC}$, leading to a decrease in the ISC rates of the monomer by 1 to 2 orders of magnitude, which is more pronounced for the polar acetonitrile than for the nonpolar toluene (Table 1). This finding represents an example of how the aggregate suppresses triplet formation.

This TDDFT analysis does not claim that the structures of the experimentally observed aggregates of $\mathbf{2}$ and $\mathbf{3}$ are dimers. In fact, the concentration dependence of the aggregate fluorescence of $\mathbf{3}$ in low-polarity solvents (Figure 2e) indicates the presence of a mixture of multimers. Furthermore, TA spectroscopy reveals transitions involving two transients in the singlet manifold ascribed as $\mathrm{S}_{1}{ }^{(\mathrm{LE})}$ and $\mathrm{S}_{1}{ }^{(\mathrm{CT})}$ (Figure 4a,b). The TDDFT optimized $\mathrm{S}_{1}$ states of the dimers have a CT character larger than that of the $\mathrm{S}_{0}$ ones. Also, the TDDFT-calculated radiative-decay rate constants, $\mathrm{k}_{\mathrm{f}}{ }^{(\mathrm{DFT})}$, for the dimers are considerably smaller than the $\mathrm{k}_{\mathrm{f}}$ values obtained from the spectroscopy analysis (Table 1). While these considerations suggest that the theoretically optimized $\mathrm{S}_{1}$ states are representative of the experimentally observed $\mathrm{S}_{1}{ }^{(\mathrm{CT})}$ ones, such an assignment warrants a great degree of caution because of the lack of evidence that the aggregates of $\mathbf{2}$ and $\mathbf{3}$ are actually dimers. Nevertheless, interrogating structures with the smallest degree of aggregation, i.e., dimers, reveals key trends about the effects of aggregation on the photophysics of these $\mathrm{NN}$ derivatives that are consistent with the experimental TA observations.

For TA spectroscopy, the concentration of the $\mathrm{NN}$ samples exceeds $0.5 \mathrm{mM}$ in order to attain sufficient optical density at the excitation wavelength for the $2 \mathrm{~mm}$ optical path length. Such high concentrations warrant attributing the trends revealed by the TA dynamics to aggregates of the NN derivatives. Changing the solvents can alter the degree of aggregation and the optical properties of the probed samples. Do the observed TA trends, therefore, originate from effects of medium polarity or from solvent-induced changes in the degree of aggregation? While converting dimers to larger multimers will affect the excited-state dynamics of these compounds, we focus on kinetic trends that span over orders of magnitude, for which the degree of aggregation might not be plausible to account. Furthermore, the TDDFT analysis of the dimers of $\mathbf{2}$ and $\mathbf{3}$ agree well with the experimentally obtained trends that correlate with solvent polarity.

The TDDFT analysis shows that the polarity-induced quenching of the monomer fluorescence of $\mathbf{2}$ results from enhancement of ISC rates. For $(\mathbf{2})_{2}$, on the other hand, IC appears to account for the decrease in its $\mathrm{S}_{1}$ lifetime in polar media. The TA spectra of $\mathbf{2}$ (representative of its aggregate) do not show the formation of triplets in any of the solvents (Figure 4a). This experimental result is consistent with efficient $\mathrm{S}_{1} \rightarrow \mathrm{S}_{0}$ IC and with the TDDFT findings for $(\mathbf{2})_{2}$ (Table 1, Figure $5 b$ ). 
The TDDFT analysis also reveals that the monomer of 3 undergoes ISC with rates on the order of $10^{11} \mathrm{~s}^{-1}$ that are practically the same for the acetonitrile and toluene PCM (Table 1). Conversely, the computed ISC rate constant for the dimer of $\mathbf{3}$, i.e., $(\mathbf{3})_{2}$, is about $6 \times 10^{10}$ $\mathrm{s}^{-1}$ for toluene and decreases by an order of magnitude when we change the solvation media to acetonitrile (Table 1). The results for $(\mathbf{3})_{2}$ are consistent with the TA dynamics of $\mathbf{3}$, which is representative of its aggregate form. The relative TA amplitude of the formed long-lived triplet decreases with an increase in solvent polarity (Figure 4b). This trend is consistent with a polarity-induced decrease in the ISC rates and an increase in the IC rates.

Medium polarity, along with the electron-donating strength of a substituent and its position relative to the nitro group, governs the deactivation dynamics of $S_{1}$. Even a weakly electrondonating group at position 5 induces sufficient $C T$ character in the $S_{1}$ state to suppress its SOC with the $T_{j}$ states and slow down ISC, e.g., from 100 fs for $\mathbf{1}$ to hundreds of picoseconds for 3 . Increasing the electron-donating strength of the group at position 5 and aggregation further slows down ISC. Strong electron-donating groups also lower the $\mathrm{S}_{1}{ }^{(\mathrm{CT})}$ state sufficiently close to the potential-energy surface of $\mathrm{S}_{0}$ and provide pathways for efficient IC. ${ }^{20}$ Placing an electron-donating group at position 4, while opening pathways for IC, does not efficiently suppress ISC. ${ }^{43}$ These findings illustrate the multifaceted complexity of how nitro groups affect emissive singlet-excited states. These trends reveal key paradigms for broadly implementable molecular designs of electron-deficient, i.e., $n$-type, organic sensitizers and potent photo-oxidants.

\section{Supplementary Material}

Refer to Web version on PubMed Central for supplementary material.

\section{ACKNOWLEDGMENTS}

This research was funded by the USA National Science Foundation (grant number CHE 1800602 and AGEP supplement fellowship for J.A.C.), the American Chemical Society Petroleum Research Fund (grant number 60651-ND4), and the USA National Institutes of Health, National Eye Institute (grant R01 EY027440). G.V.B. extends his gratitude to the Swedish Research Council for their financial support (Starting Grant No. 2020-04600). The quantum-chemical calculations were performed with computational resources provided by Swedish National Infrastructure for Computing (SNIC 2020-3-29) at the High-Performance Computing Center North (HPC2N) partially funded by the Swedish Research Council through the Grant Agreement No. 2018-05973.

\section{REFERENCES}

(1). Stolar M; Baumgartner T Organic n-type materials for charge transport and charge storage applications. Phys. Chem. Chem. Phys 2013, 15 (23), 9007-9024. [PubMed: 23674178]

(2). Yue Q; Liu W; Zhu X n-Type Molecular Photovoltaic Materials: Design Strategies and Device Applications. J. Am. Chem. Soc 2020, 142 (27), 11613-11628. [PubMed: 32460485]

(3). Wilson TM; Tauber MJ; Wasielewski MR Toward an n-Type Molecular Wire: Electron Hopping within Linearly Linked Perylenediimide Oligomers. J. Am. Chem. Soc 2009, 131 (25), 89528957. [PubMed: 19507855]

(4). Schuster DI; Cheng P; Jarowski PD; Guldi DM; Luo C; Echegoyen L; Pyo S; Holzwarth AR; Braslavsky SE; Williams RM; et al. Design, Synthesis, and Photophysical Studies of a PorphyrinFullerene Dyad with Parachute Topology; Charge Recombination in the Marcus Inverted Region. J. Am. Chem. Soc 2004, 126 (23), 7257-7270. [PubMed: 15186163] 
(5). Lu H; Bao D; Penchev M; Ghazinejad M; Vullev VI; Ozkan CS; Ozkan M Pyridine-coated lead sulfide quantum dots for polymer hybrid photovoltaic devices. Adv. Sci. Lett 2010, 3 (2), 101109.

(6). Ashcraft A; Liu K; Mukhopadhyay A; Paulino V; Liu C; Bernard B; Husainy D; Phan T; Olivier J-H A Molecular Strategy to Lock-in the Conformation of a Perylene Bisimide-Derived Supramolecular Polymer. Angew. Chem., Int. Ed 2020, 59 (19), 7487-7493.

(7). Orłowski R; Clark JA; Derr JB; Espinoza EM; Mayther MF; Staszewska-Krajewska O; Winkler JR; Jędrzejewska H; Szumna A; Gray HB; et al. Role of intramolecular hydrogen bonds in promoting electron flow through amino acid and oligopeptide conjugates. Proc. Natl. Acad. Sci. U. S. A 2021, 118 (11), No. e2026462118.

(8). Purc A; Espinoza EM; Nazir R; Romero JJ; Skonieczny K; Jeżewski A; Larsen JM; Gryko DT; Vullev VI Gating That Suppresses Charge Recombination-The Role of Mono-N-Arylated Diketopyrrolopyrrole. J. Am. Chem. Soc 2016, 138 (39), 12826-12832. [PubMed: 27617743]

(9). Krzeszewski M; Espinoza EM; Cervinka C; Derr JB; Clark JA; Borchardt D; Beran GJO; Gryko DT; Vullev VI Dipole Effects on Electron Transfer are Enormous. Angew. Chem., Int. Ed 2018, 57 (38), 12365-12369.

(10). Espinoza EM; Xia B; Darabedian N; Larsen JM; Nunez V; Bao D; Mac JT; Botero F; Wurch M; Zhou F; Vullev VI; et al. Nitropyrene Photoprobes: Making Them, and What Are They Good For? Eur. J. Org. Chem 2016, 2016, 343-356.

(11). Bao D; Upadhyayula S; Larsen JM; Xia B; Georgieva B; Nunez V; Espinoza EM; Hartman JD; Wurch M; Chang A; et al. Dipole-Mediated Rectification of Intramolecular Photoinduced Charge Separation and Charge Recombination. J. Am. Chem. Soc 2014, 136 (37), 12966-12973. [PubMed: 25162490]

(12). Espinoza EM; Clark JA; Derr JB; Bao D; Georgieva B; Quina FH; Vullev VI How Do Amides Affect the Electronic Properties of Pyrene? ACS Omega 2018, 3 (10), 12857-12867. [PubMed: 31458010]

(13). Watson AT; Matsen FA The Relation of the Frequency of Ultraviolet Absorption to the Reduction Potential of Aromatic Hydrocarbons. J. Chem. Phys 1950, 18 (9), 1305-1306.

(14). Jones G II; Lu LN; Vullev V; Gosztola D; Greenfield S; Wasielewski M Photoactive peptides. 6. Photoinduced electron transfer for pyrenesulfonamide conjugates of tryptophan-containing peptides. Mitigation of fluoroprobe behavior in N-terminal labeling experiments. Bioorg. Med. Chem. Lett 1995, 5 (20), 2385-2390.

(15). Ottonelli M; Piccardo M; Duce D; Thea S; Dellepiane G Tuning the Photophysical Properties of Pyrene-Based Systems: A Theoretical Study. J. Phys. Chem. A 2012, 116 (1), 611-630. [PubMed: 22103241]

(16). Jones G II; Vullev VI Photoinduced Electron Transfer between Non-Native Donor-Acceptor Moieties Incorporated in Synthetic Polypeptide Aggregates. Org. Lett 2002, 4 (23), 4001-4004. [PubMed: 12423071]

(17). Mewes JM; Jovanovic V; Marian CM; Dreuw A On the molecular mechanism of non-radiative decay of nitrobenzene and the unforeseen challenges this simple molecule holds for electronic structure theory. Phys. Chem. Chem. Phys 2014, 16 (24), 12393-12406. [PubMed: 24827580]

(18). Yang M; Zhang TS; Xue JD; Zheng XM Ab Initio Study of Decay Dynamics of 1Nitronaphthalene Initiated from the $\mathrm{S} 2\left(\pi \pi \mathrm{i}^{*}+\mathrm{n}(\mathrm{NO}) \pi^{*}\right)$ State. J. Phys. Chem. A 2018,122 (10), 2732-2738. [PubMed: 29488758]

(19). Plaza-Medina EF; Rodriguez-Cordoba W; Morales-Cueto R; Peon J Primary Photochemistry of Nitrated Aromatic Compounds: Excited-State Dynamics and NO Dissociation from 9Nitroanthracene. J. Phys. Chem. A 2011, 115 (5), 577-585. [PubMed: 21214230]

(20). Poronik YM; Baryshnikov GV; Deperasinska I; Espinoza EM; Clark JA; Agren H; Gryko DT; Vullev VI Deciphering the Enigma of Unusual Fluorescence in Weakly Coupled Bisnitropyrrolo[3,2-b]pyrroles. Commun. Chem 2020, 3, 190.

(21). Chen MC; Chen DG; Chou PT Fluorescent Chromophores Containing the Nitro Group: Relatively Unexplored Emissive Properties. ChemPlusChem 2021, 86 (1), 11-27. [PubMed: 33094565] 
(22). Zobel JP; Nogueira JJ; Gonzalez L Mechanism of Ultrafast Intersystem Crossing in 2Nitronaphthalene. Chem. - Eur. J 2018, 24 (20), 5379-5387. [PubMed: 29377370]

(23). Grabowski ZR; Rotkiewicz K; Rettig W Structural changes accompanying intramolecular electron transfer: Focus on twisted intramolecular charge-transfer states and structures. Chem. Rev 2003, 103 (10), 3899-4031. [PubMed: 14531716]

(24). Lopez-Arteaga R; Stephansen AB; Guarin CA; Solling TI; Peon J The Influence of Push-Pull States on the Ultrafast Intersystem Crossing in Nitroaromatics. J. Phys. Chem. B 2013, 117 (34), 9947-9955. [PubMed: 23906388]

(25). Benson S; Fernandez A; Barth ND; de Moliner F; Horrocks MH; Herrington CS; Abad JL; Delgado A; Kelly L; Chang Z; et al. SCOTfluors: Small, Conjugatable, Orthogonal, and Tunable Fluorophores for In Vivo Imaging of Cell Metabolism. Angew. Chem., Int. Ed 2019, 58 (21), 6911-6915.

(26). Ghosh PB; Whitehouse MW 7-Chloro-4-Nitrobenzo-2-Oxa-1,3-Diazole: A New Fluorigenic Reagent for Amino Acids and Other Amines. Biochem. J 1968, 108 (1), 155-156. [PubMed: 5657448]

(27). Mohammed OF; Vauthey E Excited-state dynamics of nitroperylene in solution: Solvent and excitation wavelength dependence. J. Phys. Chem. A 2008, 112 (17), 3823-3830. [PubMed: 18366202]

(28). Sinha HK; Yates K Ground-State and Excited-State Dipole-Moments of Some Nitroaromatics: Evidence for Extensive Charge-Transfer in Twisted Nitrobenzene Systems. J. Chem. Phys 1990, 93 (10), 7085-7093.

(29). Sadowski B; Kaliszewska M; Poronik YM; Czichy M; Janasik P; Banasiewicz M; Mierzwa D; Gadomski W; Lohrey TD; Clark JA et al. Potent strategy towards strongly emissive nitroaromatics through a weakly electron-deficient core Chem. Sci 2021, DOI: 10.1039/ D1SC03670J.

(30). Morales-Cueto R; Esquivelzeta-Rabell M; Saucedo-Zugazagoitia J; Peon J Singlet excited-state dynamics of nitropolycyclic aromatic hydrocarbons: Direct measurements by femto-second fluorescence up-conversion. J. Phys. Chem. A 2007, 111 (4), 552-557. [PubMed: 17249742]

(31). Zugazagoitia JS; Almora-Diaz CX; Peon J Ultrafast intersystem crossing in 1-nitronaphthalene. An experimental and computational study. J. Phys. Chem. A 2008, 112 (3), 358-365. [PubMed: 18166024]

(32). Corkill JM; Graham-Bryce IJ The luminescence of some substituted naphthalenes. J. Chem. Soc 1961, 3893.

(33). Ermolaev VL; Svitashev KK Phosphorescence and fluorescence quantum output of some 1derivatives of naphthalene solutions at $-196^{\circ}$. Opt. Spektrosk 1959, 7, 664.

(34). Nielsen AT; DeFusco AA; Browne TE Nitration of bis(amido)naphthalenes. J. Org. Chem 1985, 50 (22), 4211-4218.

(35). Hachiya S; Asai K; Konishi G.-i. Unique solvent-dependent fluorescence of nitro-groupcontaining naphthalene derivatives with weak donor-strong acceptor system. Tetrahedron Lett 2013, 54 (14), 1839-1841.

(36). Rodriguez JG; Tejedor JL Carbon Networks Based on 1,5-Naphthalene Units. Synthesis of 1,5-Naphthalene Nanostructures with Extended $\pi$-Conjugation. J. Org. Chem 2002, 67 (22), 7631-7640. [PubMed: 12398483]

(37). Singh AK; Darshi M; Kanvah S a, $\omega$-Diphenylpolyenes Cabable of Exhibiting Twisted Intramolecular Charge Transfer Fluorescence: A Fluorescence and Fluorescence Probe Study of Nitro- and Nitrocyano-Substituted 1,4-Diphenylbutadienes. J. Phys. Chem. A 2000, 104 (3), 464-471.

(38). Upadhyayula S; Nunez V; Espinoza EM; Larsen JM; Bao D; Shi D; Mac JT; Anvari B; Vullev VI Photoinduced dynamics of a cyanine dye: parallel pathways of non-radiative deactivation involving multiple excited-state twisted transients. Chem. Sci 2015, 6, 2237-2251. [PubMed: 29449923]

(39). Milne PH; Wayner DDM; Decosta DP; Pincock JA Substituent and Charge-Distribution Effects on the Redox Potentials of Radicals: Thermodynamics for Homolytic Versus Heterolytic Cleavage in the 1-Naphthylmethyl System. Can. J. Chem 1992, 70 (1), 121-127. 
(40). Matsunaga A; Yasuhara A Complete dechlorination of 1-chloronaphthalene by electrochemical reduction with naphthalene radical anion as mediator. Environ. Sci. Technol 2003, 37 (15), 34353441. [PubMed: 12966992]

(41). Fontanesi C; Borsari M; Andreoli R; Benedetti L; Grandi G; Battistuzzi Gavioli G ElectrochemicalBehaviour of $N$-Tosylglycine and $N$-Dansylglycine in Several Solvents: The Role of the $\mathrm{RSO}_{2}$-Groups on the Physicochemical Properties of Glycine. Electrochim. Acta 1989, 34 (6), 759-765.

(42). Pospíšil P; Luxem KE; Ener M; Sýkora J; Kocábová J; Gray HB; Vlček A; Hof M Fluorescence Quenching of (Dimethylamino)naphthalene Dyes Badan and Prodan by Tryptophan in Cytochromes P450 and Micelles. J. Phys. Chem. B 2014, 118 (34), 10085-10091. [PubMed: 25079965]

(43). Collado-Fregoso E; Zugazagoitia JS; Plaza-Medina EF; Peon J Excited-State Dynamics of Nitrated Push-Pull Molecules: The Importance of the Relative Energy of the Singlet and Triplet Manifolds. J. Phys. Chem. A 2009, 113 (48), 13498-13508. [PubMed: 19839627]

(44). Niko Y; Didier P; Mely Y; Konishi G; Klymchenko AS Bright and photostable push-pull pyrene dye visualizes lipid order variation between plasma and intracellular membranes. Sci. Rep 2016, 6, 18870. [PubMed: 26750324]

(45). Suhaj A; Le Marois A; Williamson DJ; Suhling K; Lorenz CD; Owen DM PRODAN differentially influences its local environment. Phys. Chem. Chem. Phys 2018, 20 (23), 1606016066. [PubMed: 29850681]

(46). Chen T; Lee SW; Abelt CJ 1,5-Prodan Emits from a Planar Intramolecular Charge-Transfer Excited State. ACS Omega 2018, 3 (5), 4816-4823. [PubMed: 31458698]

(47). Seiler N Use of the Dansyl reaction in biochemical analysis. Methods Biochem. Anal 2006, 18, 259-337.

(48). Walker JM The Dansyl-Edman Method for Peptide Sequencing. Methods Mol. Biol 1994, 32, 329-334. [PubMed: 7951733]

(49). Figueira-Duarte TM; Müllen K Pyrene-Based Materials for Organic Electronics. Chem. Rev 2011, 111 (11), 7260-7314. [PubMed: 21740071]

(50). Bao D; Millare B; Xia W; Steyer BG; Gerasimenko AA; Ferreira A; Contreras A; Vullev VI Electrochemical Oxidation of Ferrocene: A Strong Dependence on the Concentration of the Supporting Electrolyte for Nonpolar Solvents. J. Phys. Chem. A 2009, 113 (7), 1259-1267. [PubMed: 19199684]

(51). Espinoza EM; Clark JA; Soliman J; Derr JB; Morales M; Vullev VI Practical Aspects of Cyclic Voltammetry: How to Estimate Reduction Potentials When Irreversibility Prevails. J. Electrochem. Soc 2019, 166 (5), H3175-H3187.

(52). Angulo G; Grampp G; Rosspeintner A Recalling the appropriate representation of electronic spectra. Spectrochim. Acta, Part A 2006, 65A (3-4), 727-731.

(53). Hodgson HH; Hathway DE Absorption spectra of some mononitronaphthylamines, with observations on their structures. II. 3-, 5-, 6-, 7-, and 8-nitro-2-naphthylamines and 6-nitro-1naphthyl-amine. Trans. Faraday Soc 1947, 43, 643-648.

(54). Reichardt C; Vogt RA; Crespo-Hernandez CE On the origin of ultrafast nonradiative transitions in nitro-polycyclic aromatic hydrocarbons: Excited-state dynamics in 1-nitronaphthalene. J. Chem. Phys 2009, 131 (22), 224518/1-224518/15. [PubMed: 20001068]

(55). Zugazagoitia JS; Collado-Fregoso E; Plaza-Medina EF; Peon J Relaxation in the Triplet Manifold of 1-Nitronaphthalene Observed by Transient Absorption Spectroscopy. J. Phys. Chem. A 2009, 113 (5), 805-810. [PubMed: 19140711]

(56). Upadhyayula S; Bao D; Millare B; Sylvia SS; Habib KMM; Ashraf K; Ferreira A; Bishop S; Bonderer R; Baqai S; et al. Permanent Electric Dipole Moments of Carboxyamides in Condensed Media: What Are the Limitations of Theory and Experiment? J. Phys. Chem. B 2011, 115 (30), 9473-9490. [PubMed: 21682315]

(57). Vogt RA; Reichardt C; Crespo-Hernandez CE Excited-State Dynamics in Nitro-Naphthalene Derivatives: Intersystem Crossing to the Triplet Manifold in Hundreds of Femtoseconds. J. Phys. Chem. A 2013, 117 (30), 6580-6588. [PubMed: 23845162] 
(58). Orozco-Gonzalez Y; Coutinho K; Peon J; Canuto S Theoretical study of the absorption and nonradiative deactivation of 1-nitronaphthalene in the low-lying singlet and triplet excited states including methanol and ethanol solvent effects. J. Chem. Phys 2012, 137 (5), 054307/1054307/8. [PubMed: 22894348]

(59). Yang M; Zhang T; Xue J; Zheng X Ab Initio Study of Decay Dynamics of 1-Nitronaphthalene Initiated from the S2 $\left(\pi \pi^{*}+\right.$ nNO $\left.\pi^{*}\right)$ State. J. Phys. Chem. A 2018, 122 (10), 2732-2738.

[PubMed: 29488758] 

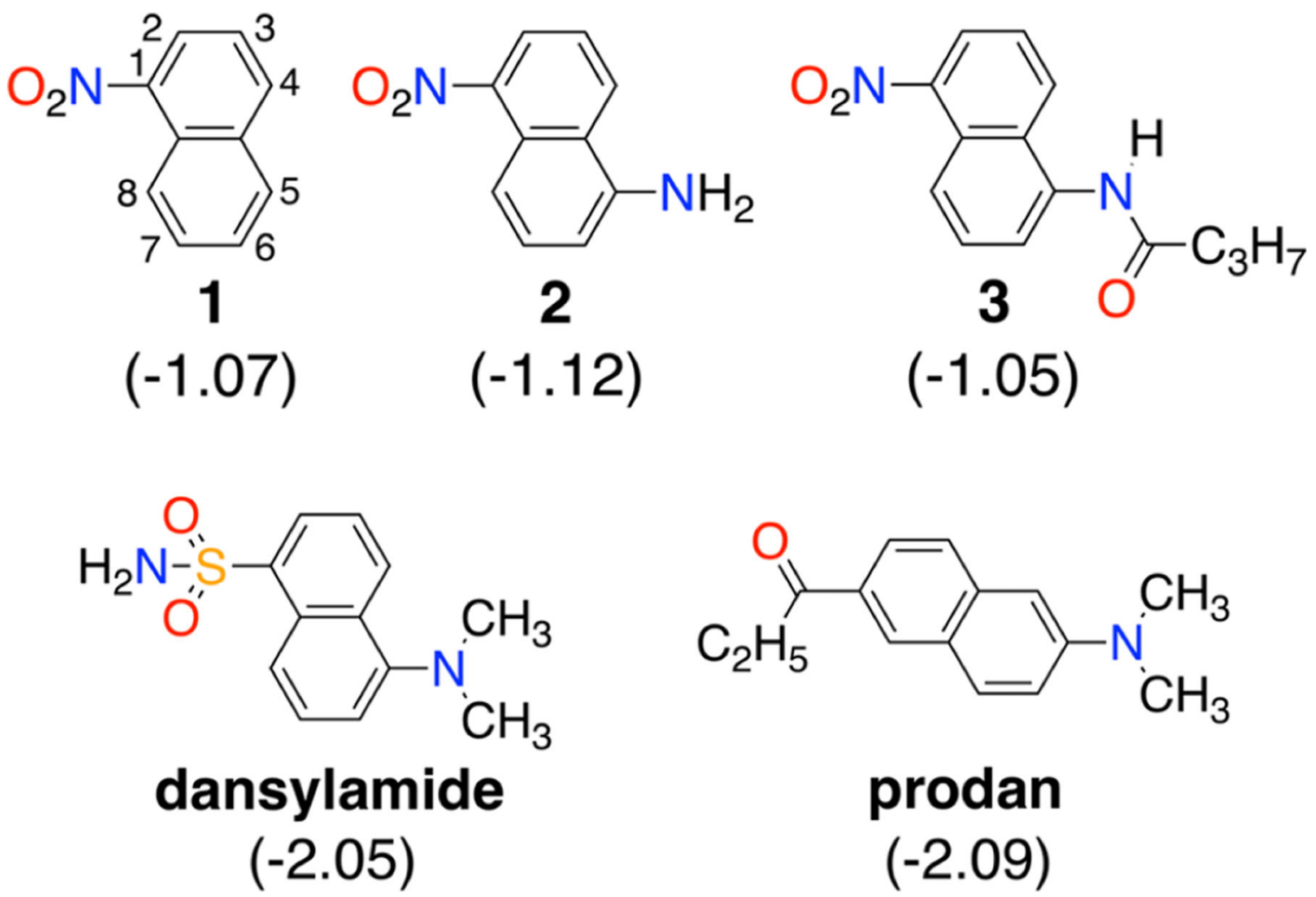

Chart 1. Nitronaphthalenes with Electron-Donating Groups, along with Examples of Broadly Used Push-Pull Naphthalene Dyes ${ }^{a}$

aThe reduction potentials of $\mathbf{1}, \mathbf{2}$, and $\mathbf{3}, E_{\mathrm{XIX}}^{(1 / 2)} \bullet-/ V$ vs SCE, measured for acetonitrile (Figure 1), are listed in the parentheses. For naphthalene and 1-methylnaphthalene, $E_{\mathrm{XI} X}^{(1 / 2)} \bullet-\approx-2.5 \quad \mathrm{~V}$ vs SCE; ${ }^{39,40}$ for dansylamide, $E_{\mathrm{XIX}}^{(1 / 2)} \bullet-\approx-2.05$ V vs SCE; ${ }^{41}$ and for prodan, $E_{\mathrm{XIX}}^{(1 / 2)} \bullet-\approx-2.09 \mathrm{~V}$ vs SCE. ${ }^{42}$ 


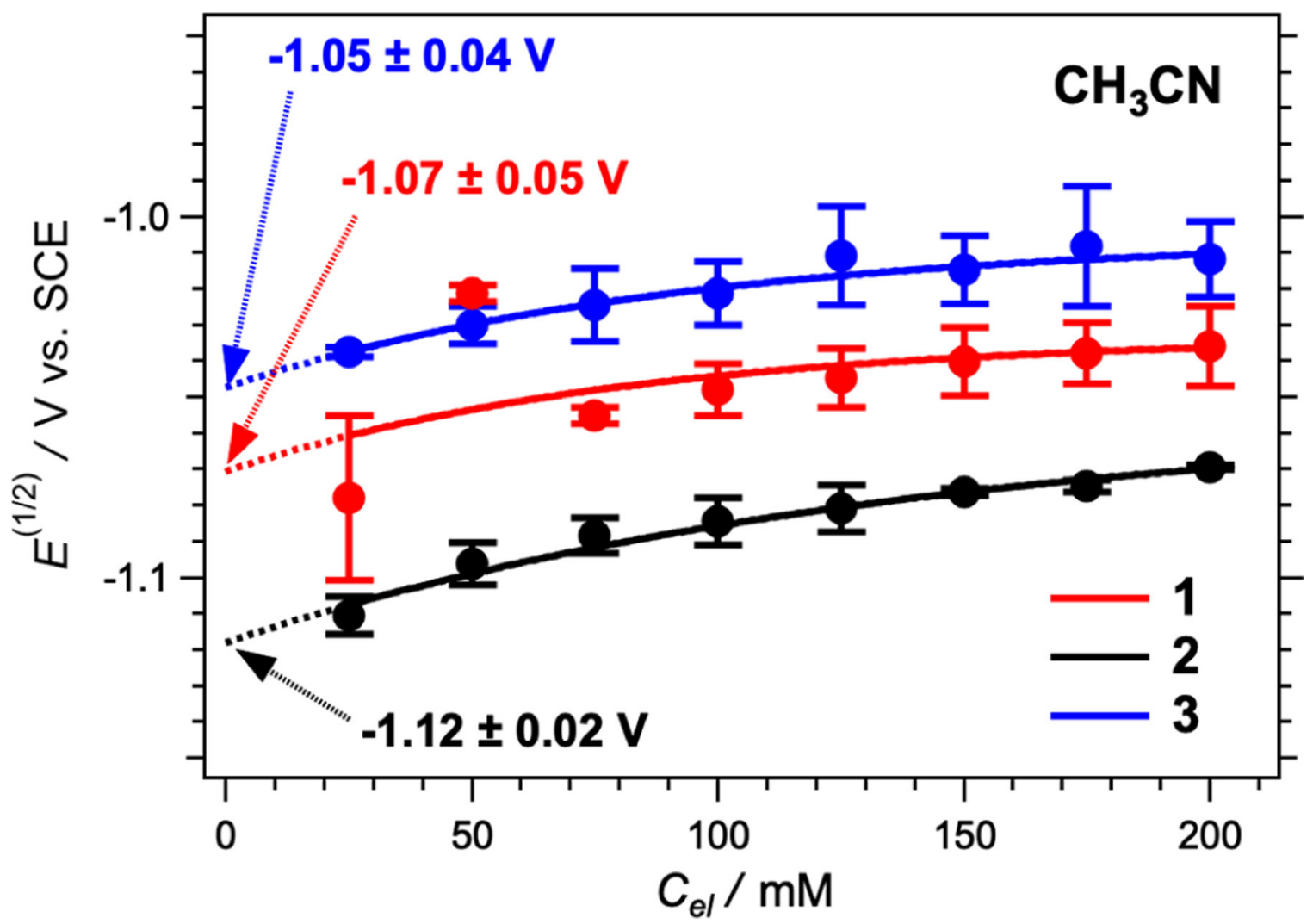

Figure 1.

Dependence of the half-wave potentials, $E^{(1 / 2)}$, of $\mathbf{1}, \mathbf{2}$, and $\mathbf{3}$ (extracted from their cyclic voltammograms) on the electrolyte concentration, $C_{\mathrm{el}}$, in $\mathrm{CH}_{3} \mathrm{CN}$. The supporting electrolyte is $\mathrm{N}\left(n-\mathrm{C}_{4} \mathrm{H}_{9}\right)_{4} \mathrm{PF}_{6}$. The dotted lines represent extrapolations to $C_{\mathrm{el}}=0$ yield estimates of $E^{(1 / 2)}$ of $\mathbf{1}, \mathbf{2}$, and $\mathbf{3}$ for neat acetonitrile. ${ }^{50,51}$ 

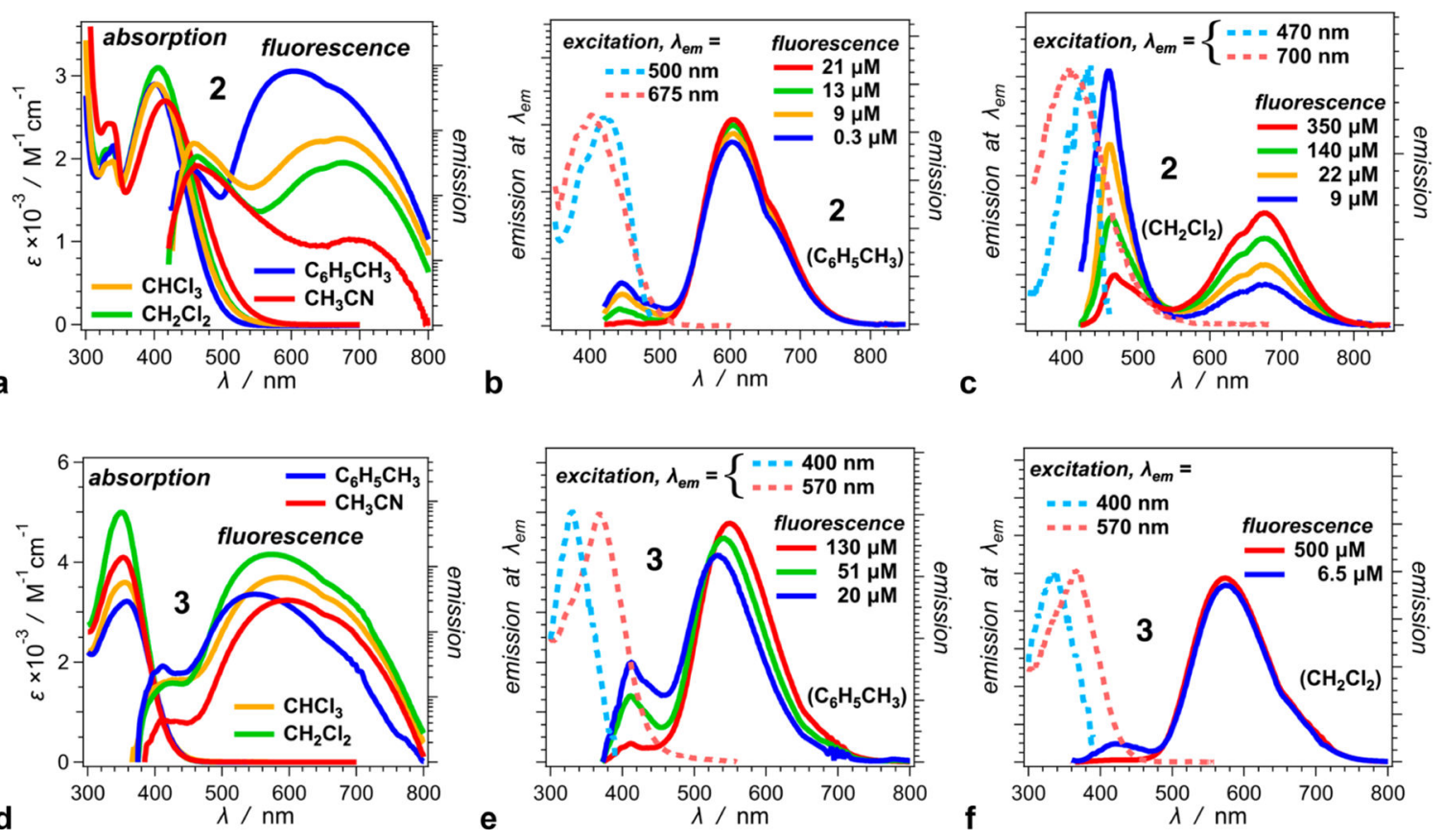

Figure 2.

Optical absorption and fluorescence of 2 and 3. (a,d) Absorption and emission spectra for different solvents. The fluorescence is divided by $\left(1-10^{-A\left(\lambda_{e x}\right)}\right)$; thus, the spectra have areas under them proportional to $\phi_{\mathrm{f}}$, and they are plotted in logarithmic scales for improved visualization. The concentrations of $2, \mathrm{C}(2)$, are $18 \mu \mathrm{M}$ for toluene, $140 \mu \mathrm{M}$ for $\mathrm{CHCl}_{3}$ and $\mathrm{CH}_{2} \mathrm{Cl}_{2}$, and $150 \mu \mathrm{M}$ for $\mathrm{CH}_{3} \mathrm{CN}$, and the concentrations of $\mathbf{3}, \mathrm{C}(\mathbf{3})$, are $130 \mu \mathrm{M}$ for toluene and $\mathrm{CHCl}_{3}, 510 \mu \mathrm{M}$ for $\mathrm{CH}_{2} \mathrm{Cl}_{2}$, and $180 \mu \mathrm{M}$ for $\mathrm{CH}_{3} \mathrm{CN}$. (b,c,e,f) Concentration dependence of the fluorescence spectra (with normalized areas), along with the excitation spectra recorded at the short-wavelength and long-wavelength bands ascribed to emission from monomers and aggregates (for $2, \lambda_{\mathrm{ex}}=410 \mathrm{~nm}$; and for $\mathbf{3}, \lambda_{\mathrm{ex}}=350 \mathrm{~nm}$ ). 

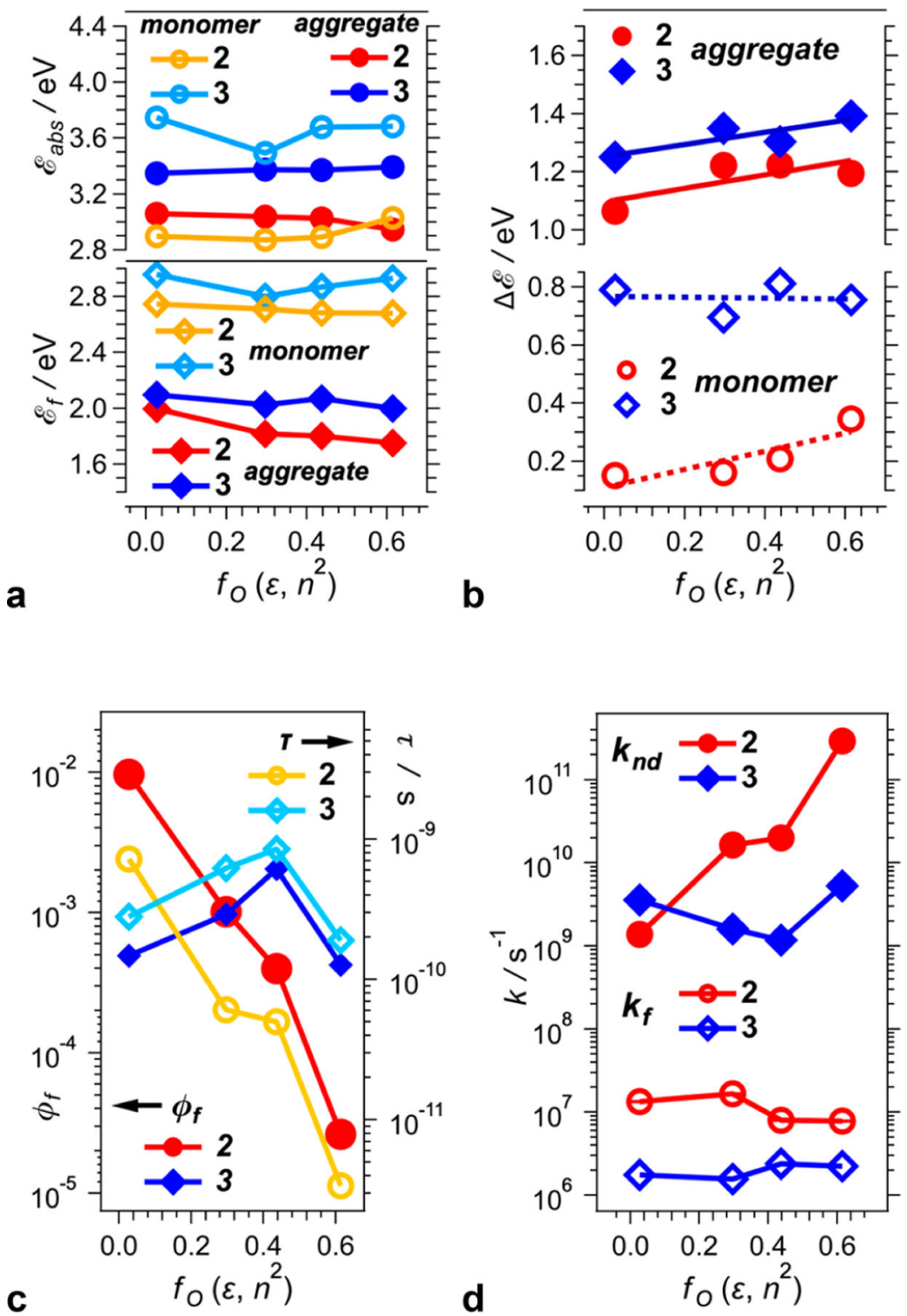

Figure 3.

Dependence of excited-state properties of $\mathbf{2}$ and $\mathbf{3}$ on solvent polarity as represented by the Onsager function: $f_{\mathrm{O}}(x)=2(x-1) /(2 x+1), f_{\mathrm{O}}\left(\varepsilon, n^{2}\right)=f_{\mathrm{O}}(\varepsilon)-f_{\mathrm{O}}\left(n^{2}\right)$. (a) Energies of the absorption maxima, $\boldsymbol{\varepsilon}_{\mathrm{abs}}$, obtained from absorption and excitation spectra, and fluorescence maxima, $\boldsymbol{\varepsilon}_{\mathrm{f}}$, from emission spectra. (b) Stokes' shifts, $\Delta \boldsymbol{\varepsilon}=\boldsymbol{\varepsilon}_{\mathrm{abs}}-\boldsymbol{\varepsilon}_{\mathrm{f}}$. (c) Fluorescence quantum yields, $\phi_{\mathrm{f}}$, and $\mathrm{S}_{1}$ lifetimes, $\boldsymbol{\tau}$, for the aggregated species that dominate the photophysics. (d) Rate constants of the radiative, $k_{\mathrm{f}}$, and nonradiative, $k_{\mathrm{nd}}$, decays. 

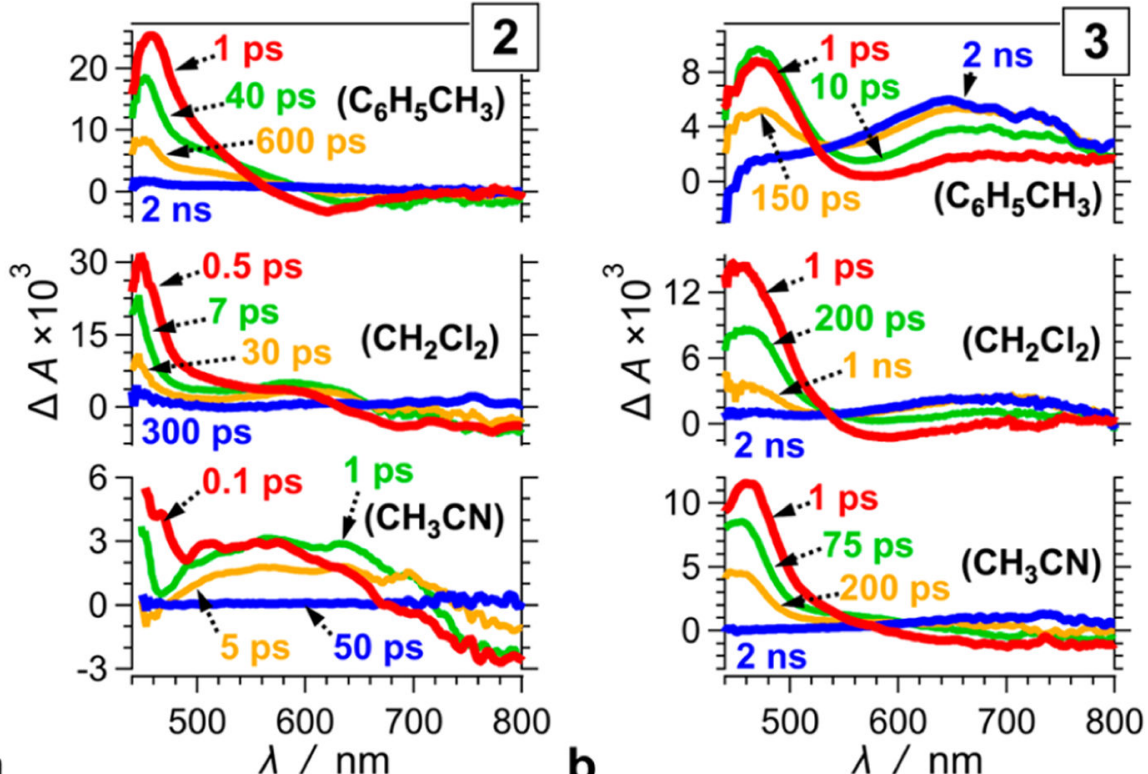

a $\lambda / \mathrm{nm}$
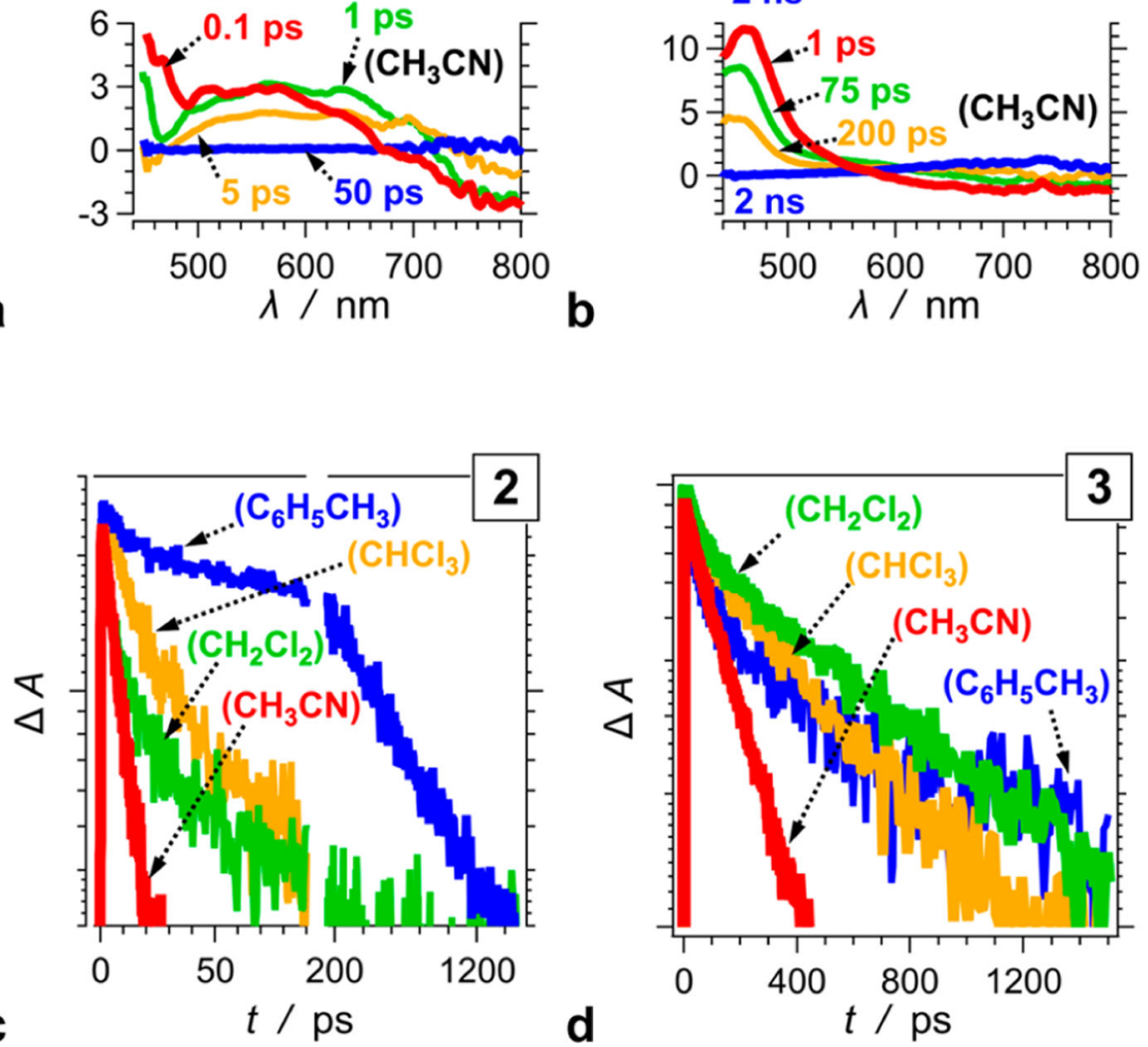

Figure 4.

TA dynamics of $\mathbf{2}$ and $\mathbf{3}$ in solvents with different polarity ( $\lambda_{\mathrm{ex}}=400 \mathrm{~nm}, 4 \mu \mathrm{J}$ per pulse). TA spectra of (a) $2(700 \mu \mathrm{M})$ and (b) $3(2 \mathrm{mM})$. TA decays of the $\mathrm{S}_{1}$ states (with ordinates in logarithmic scales) of (c) 2, recorded at $454 \mathrm{~nm}$ for toluene, $450 \mathrm{~nm}$ for $\mathrm{CHCl}_{3}$ and $\mathrm{CH}_{2} \mathrm{Cl}_{2}$, and $630 \mathrm{~nm}$ for $\mathrm{CH}_{3} \mathrm{CN}$, and (d) 3 , recorded at $450 \mathrm{~nm}$ for toluene and $\mathrm{CHCl}_{3}$ and $460 \mathrm{~nm}$ for $\mathrm{CH}_{2} \mathrm{Cl}_{2}$ and $\mathrm{CH}_{3} \mathrm{CN}$. 


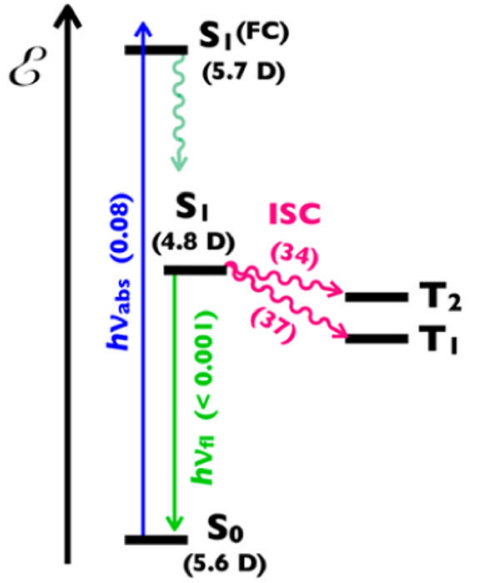

a

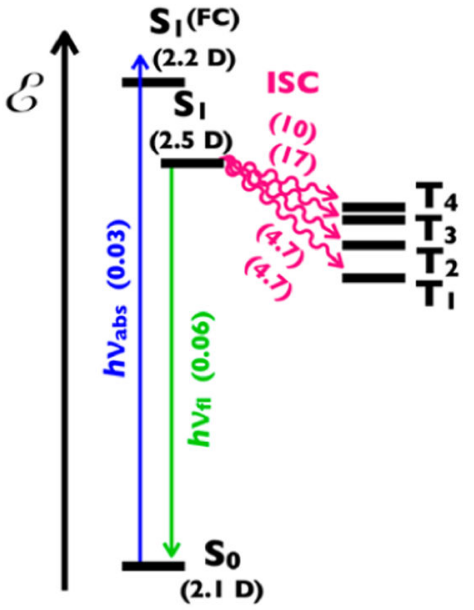

b

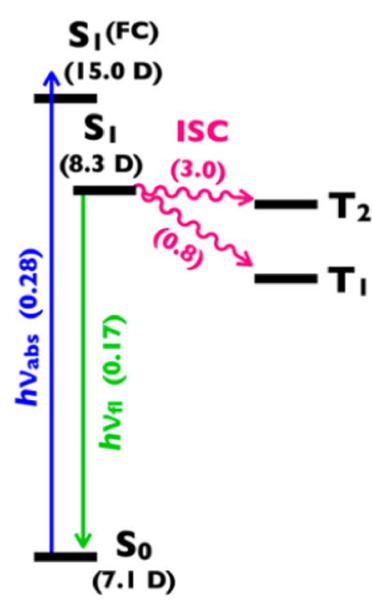

2

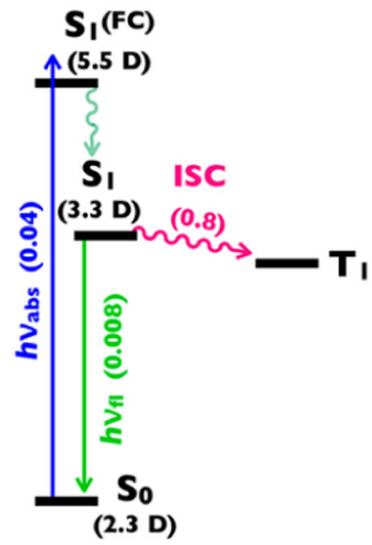

$(2)_{2}$

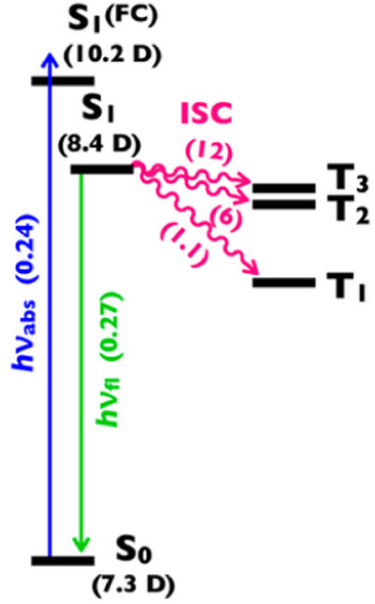

\section{3}

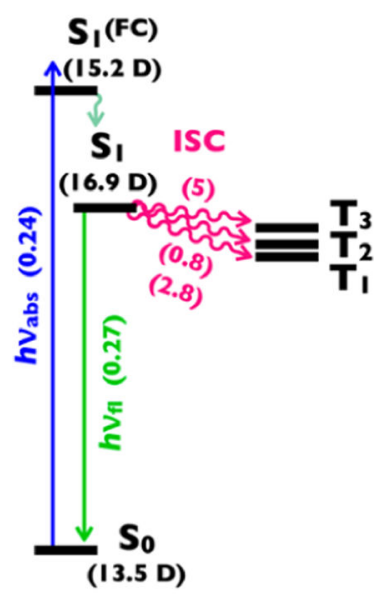

$(3)_{2}$

Figure 5.

Jablonski diagrams depicting the excited-state dynamics of (a) 1, 2, and 3 monomers and (b) their dimers, as revealed by (TDA)-TDDFT analysis at the wB97X/TZP level of theory with toluene solvent implemented within the COSMO model. Under the labels of the singlet states, their permanent electric dipoles are listed in parentheses. The oscillators strengths of the absorption, $h v_{\mathrm{abs}}$, and fluorescence, $h v_{\mathrm{fl}}$, transitions are listed in parentheses along the corresponding arrows. For each $\mathrm{S}_{1} \rightarrow \mathrm{T}_{\mathrm{j}}$ ISC transition, the value the SOC matrix element (in $\mathrm{cm}^{-1}$ ) is listed in parentheses. 
Rybicka-Jasińska et al.

Page 20

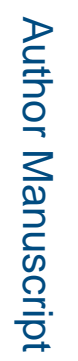



递

응

ำ

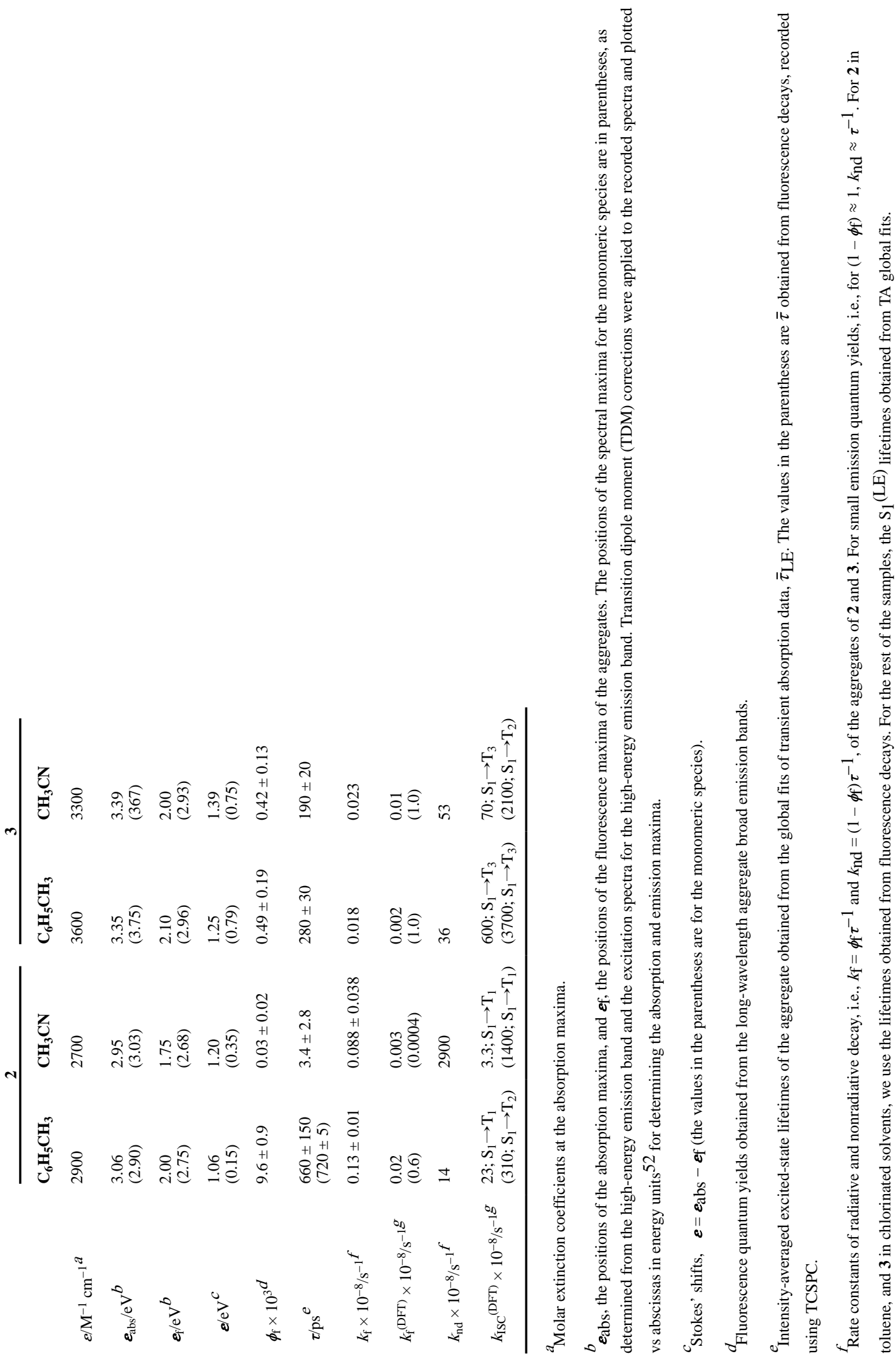

J Phys Chem Lett. Author manuscript; available in PMC 2022 January 29. 


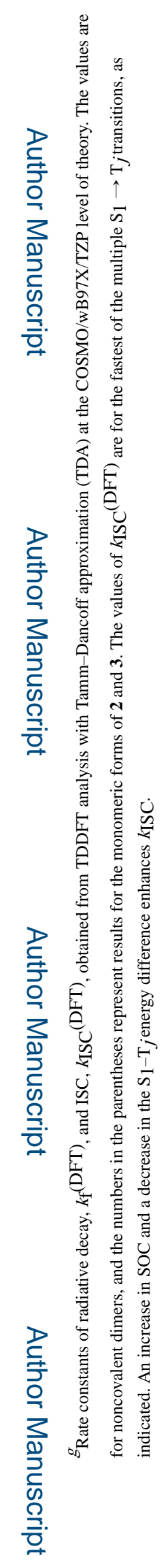

